\title{
Parkinson's Disease and Its Dermatological Associations: Is Your Skin Whispering You a Diagnosis?
}

\author{
Puja Shah ${ }^{1}$, Prem Raj Sagar ${ }^{1}$, Norah Alhumaidi ${ }^{1}$, Vijaya Chaitanya Bollampally ${ }^{2}$, Bilal Haider Malik ${ }^{1}$ \\ 1. Internal Medicine, California Institute of Behavioral Neurosciences \& Psychology, Fairfield, USA 2. Neurological \\ Surgery, California Institute of Behavioral Neurosciences \& Psychology, Fairfield, USA
}

Corresponding author: Puja Shah, peace2941@gmail.com

\begin{abstract}
Parkinson's disease (PD) is a neurodegenerative disease characterized clinically by the triad of resting tremor, rigidity, and bradykinesia. Although PD is primarily known for motor disturbance, $98.6 \%$ of patients experience one or more non-motor symptoms at all stages of the disease. Dermatological disorders are discussed as common non-motor associations of PD since the 20th century. Many studies have shown that patients of $\mathrm{PD}$ are predisposed to skin disorders. This article is a traditional review done to analyze the association between PD and its dermatological manifestations. We did a literature search using six keywords in the PubMed database and took the relevant articles published in the last 10 years. We reviewed more than 100 articles, which also included animal studies. On meticulous review, we observed an increased incidence of certain skin disorders like seborrheic dermatitis, bullous pemphigoid, rosacea, and melanoma in patients of PD. These disorders share either common risk factors or underlying mechanisms revolving around genetics, immunology, inflammation, and pathophysiology of $\mathrm{PD}$, but the exact causation yet seems obscured. We believe that this opens a horizon for more research in the link between the skin and nervous system. We also emphasize that the dermatologists, neurologists and general physicians should address the cutaneous disorders in PD timely, educate their patients, help them lessen the psychosocial distress, and improve their quality of life.
\end{abstract}

Categories: Dermatology, Internal Medicine, Neurology

Keywords: skin, parkinson's disease, melanoma, seborrheic dermatitis, bullous pemphigoid, rosacea

\section{Introduction And Background}

The second most common neurodegenerative disease is Parkinson's disease (PD) [1]. It had a worldwide burden of 2.5 million in 1990, which increased to 6.1 million in 2016. The incidence is 1.4 times more in males as compared to females after the fifth decade of life. There is an increase in the burden of PD with increasing age, decreasing smoking and improved socio-demographic index [2].

Received 07/05/2020

Review began 07/12/2020 Review ended 08/15/2020 Published 08/22/2020

() Copyright 2020 Shah et al. This is an open access article distributed under the terms of the Creative Commons Attribution License CC-BY 4.0., which permits unrestricted use, distribution, and reproduction in any medium, provided the original author and source are credited.
PD is primarily a motor disorder, but it also manifests with one or more non-motor symptoms in $98.6 \%$ of patients at all stages of the disease [3]. It is defined as a clinical triad of resting tremor, rigidity, and bradykinesia [4]. However, non-motor symptoms may also precede or appear during the disease course, along with neurological symptoms. This observation is leading to a change in the definition of PD, which no longer remains limited to the above triad [4]. The non-motor symptoms of PD include cognitive dysfunction, mood disorders, sleep disorders, gastrointestinal disorders, urinary disorders, autonomic dysfunction, and dermatological disorders [3].

There is a loss of dopaminergic neurons in the substantia nigra in PD. It explains the basis of using levodopa (L-dopa) in the treatment. Nevertheless, the lack of disease-modifying therapy shows that the understanding of PD pathogenesis is still an under-explored arena. There are several proposed theories like misfolding of alpha-synuclein, neuroinflammation, mitochondrial dysfunction, neurotransmitter driven alteration of brain neuronal networks [5]. Several genetic mutations, like Parkin (PARK2) and PTEN-induced kinase 1 (PINK1) (PARK6) genes, are also implicated in many cases [5]. There is widespread extranigral involvement all across the human body from the skin, gastrointestinal tract to the peripheral nerves in PD [3, 4].

Dermatological disorders are one of the common non-motor symptoms of PD discussed since the 20th century [5]. The associated skin problems are not recognized and are often underestimated while planning the strategy for diagnosis and treatment. This article will focus on the dermatological associations that can precede before or present during the motor symptoms of $\mathrm{PD}$, which must be addressed by the treating physician, potentially improving the patient's quality of life. Exploring the increased incidence of certain skin disorders in PD may fill the missing links in the pathogenesis of PD. This article intends to review most of the recent articles published on PubMed, which shows the relation between PD and skin disorders, and also, the references of those articles are accessed to reveal additional relevant studies. 


\section{Cureus}

\section{Review}

\section{Method}

Literature was searched in PubMed using regular keywords and Medical Subject Headings (MeSH) keywords and data collection was done. Table 1 shows regular keywords and MeSH keywords for literature search.

\begin{tabular}{|c|c|c|c|}
\hline Regular Keyword & Number of Results & MeSH Keyword & Number of Results \\
\hline Skın & 792,954 & Skın & 220,697 \\
\hline Melanoma & 132,696 & Melanoma & 93,300 \\
\hline Parkinson's disease & 110,684 & Parkinson disease & 64,695 \\
\hline Bullous Pemphigoid & 5,450 & Parkınson disease and Skın & 164 \\
\hline Rosacea & 4,005 & Parkınson disease and Melanoma & 103 \\
\hline Seborrheic dermatitis & 3,394 & & \\
\hline Parkinson's disease, Skin & 934 & & \\
\hline Parkinson's disease, Melanoma & 238 & & \\
\hline Parkinson's disease, Neurodermatology & 62 & & \\
\hline Parkinson's disease, Bullous pemphigoid & 43 & & \\
\hline Parkinson's disease, Seborrheic dermatitis & 42 & & \\
\hline Neurodermatology & 25 & & \\
\hline Parkinson's disease, Rosacea & 21 & & \\
\hline
\end{tabular}

TABLE 1: Regular keywords and MeSH keywords for literature search

MeSH: Medical Subject Headings

Studies were selected after applying the following inclusion criterion: paper published within the past 10 years. No exclusion criteria were used.

\section{Results}

Table 2 shows the total number of articles after applying inclusion criteria. 


\section{Cureus}

\begin{tabular}{|c|c|c|c|}
\hline Regular Keyword & $\begin{array}{l}\text { Number of results after inclusion } \\
\text { criterion }\end{array}$ & MeSH Keyword & $\begin{array}{l}\text { Number of results after inclusion } \\
\text { criterion }\end{array}$ \\
\hline Skıın & 269,286 & Skın & 57,449 \\
\hline Melanoma & 56,137 & Melanoma & 31,797 \\
\hline Parkinson's disease & 56,987 & Parkinson disease & 27,846 \\
\hline Bullous Pemphigoid & 1,943 & Parkinson disease and Skin & 74 \\
\hline Rosacea & 1,763 & $\begin{array}{l}\text { Parkınson disease and } \\
\text { Melanoma }\end{array}$ & 45 \\
\hline Seborrheic dermatitis & 747 & & \\
\hline Neurodermatology & 11 & & \\
\hline Parkinson's disease, Skin & 539 & & \\
\hline Parkinson's disease, Melanoma & 132 & & \\
\hline $\begin{array}{l}\text { Parkinson's disease, } \\
\text { Neurodermatology }\end{array}$ & 61 & & \\
\hline $\begin{array}{l}\text { Parkinson's disease, Bullous } \\
\text { Pemphigoid }\end{array}$ & 35 & & \\
\hline $\begin{array}{l}\text { Parkinson's disease, Seborrheic } \\
\text { dermatitis }\end{array}$ & 11 & & \\
\hline Parkinson's disease, Rosacea & 17 & & \\
\hline
\end{tabular}

TABLE 2: Total number of articles after applying inclusion criterion

MeSH: Medical Subject Headings

A cumulative of more than 100 articles were selected in writing this literature review. Additional studies were identified from manual searches of references in the retrieved articles.

\section{Discussion}

\section{Seborrheic dermatitis}

Seborrheic dermatitis (SD) is a chronic inflammatory skin disorder affecting sebum rich areas of the face, scalp, upper back, and chest, presenting predominantly as red scaly rashes. About $52-59 \%$ of PD patients, as opposed to $3 \%$ in the general population, suffer from SD leading to significant psychosocial distress [6].

Malassezia species (M. restrica and M. globosa) are the fungi present on the skin of patients of SD. The fact that antifungal works well against SD suggest that this commensal yeast has a role in the disease process. Malassezia usually proliferates in sebum rich areas. According to a study by Arsenijevic et al., PD also promotes the production of sebum on the skin [6]. The dopamine deficiency causes an inadequacy of melanocyte-stimulating hormone (MSH) inhibiting factor. This inadequacy increases the level of $\alpha-\mathrm{MSH}$, which results in a surge of sebum production. Similarly, reduced facial mobility in PD results in poor hygiene, contributing to more sebum accumulation [6-8]. This correlation gives a clue to any relationship, if present, between Malassezia and PD. A study by Kohn SR et al. shows an improvement of seborrheic dermatitis after starting L-dopa therapy in PD [9]. However, some other studies depict no such improvement [10]. A retrospective study by Taner et al. endorses SD as a premotor feature of PD. According to this study, SD increases the risk of being diagnosed with PD many years later [11]. These caveats in studies contradicting each other cannot establish Malassezia entirely as an association between SD and PD.

Many studies revolve around the role of CD4 T cells in SD, PD, and Malassezia growth. The T-helper cell (TH1) is important in the control of Malassezia and this is explained by the use of immune modulators in the treatment of SD [12]. A study by Lally A et al. shows that anti-T cell drugs (azathioprine and cyclosporine) are a major risk factor for SD [13]. Similarly, there is an association of low CD4 T cell count in PD and a weak TH1 response against Malassezia in patients of PD having concomitant SD [14,15]. Another study suggests that reduced facial mobility in PD causes a decrease in lymph flow, restricting the access of CD4 T cells to reach skin [7]. These studies can be linked by the fact that acquired immune deficiency syndrome (AIDS), 
which have weak CD4 T cell response, shows an increased risk of SD as well as increased incidence of parkinsonism in a young individual [7]. However, the response of SD to anti-retroviral therapy is variable in patients of AIDS. So we can only assume that SD and PD can be linked with weak TH1 response. More studies are required to fully support that.

PD and SD share common risk factors or underlying mechanisms but the exact pathophysiology showing one causes the other is yet to be determined. As this is a chronic inflammatory skin condition patients should be educated to focus on limiting the symptoms, rather than cure, and enhance the quality of life. Diagnosis of SD is mainly clinical and various antifungal agents, keratolytic, corticosteroids and immunomodulators can be used [12].

\section{Rosacea}

Rosacea is a benign chronic skin disorder with a multifactorial cause. There are four major clinical subtypes: erythematotelangiectatic (ETR), papulopustular (PPR), phymatous (PhR), and ocular. This inflammatory condition presents with a constellation of symptoms like flushing, persistent redness, inflammatory papules, pustules, telangiectasia, and hypertrophy of sebaceous glands of the nose. It has a prevalence of approximately $2 \%$ to $22 \%$ in the white population. As it predominantly affects the face, about $75 \%$ of rosacea patients have low self-esteem stressing the psychosocial impact of this condition [16].

In 2001 an observational study consisting of 70 PD patients showed surprising data. About $18.8 \%$ of them had rosacea, and $31.9 \%$ of them had flushing symptoms [17]. Later a large epidemiological study to examine the association between rosacea and PD was done by Egeberg alexander et al. It was a Danish cohort study done on 5.4 million adults observed for 15 years (1997-2011). There was a two-times increase in the risk of PD among patients diagnosed with rosacea than those who were not, and the incidence of PD was 2.4 years earlier than the reference population. The increased risk of PD was seen mostly in the ocular subtype of rosacea. There was a $2 \%$ decrease in the risk of PD in those taking the tetracyclines prescription regardless of rosacea diagnosis [18]. Another large retrospective study in the US using the electronic medical record of 803,005 adult patients (17,682 with rosacea diagnosis) followed over more than five years showed an increased risk of PD in rosacea patients $(\mathrm{OR}=1.39 ; 95 \% \mathrm{CI}: 1.04-1.85 ; \mathrm{p}<0.02)$.The age at rosacea diagnosis was higher in patients who ultimately got PD than those rosacea patients who did not get PD. Out of rosacea patients, those exposed to tetracycline showed no relation between rosacea and PD, whereas those not exposed to tetracyclines showed the relation [19]. Many other studies hypothesize the possible mechanisms linking the PD and rosacea [20-22].

Rosacea has up-regulation of matrix metalloproteinase (MMP) enzyme, notably MMP-1, MMP-3, MMP-9, which has a role in tissue breakdown and repair [20]. Likewise, a mice model of PD also depicts the role of MMP-1, MMP-3 in cerebrospinal fluid (CSF) in dopaminergic inflammation, and neurodegeneration [21]. Another common link to both diseases is the association with Helicobacter pylori and small intestinal bacterial overgrowth (SIBO) [22]. PPR form of rosacea has shown an association with microorganisms [22]. Furthermore, there is also evidence of aggravation of neuronal deaths in patients with long term gastrointestinal infections by these organisms [22]. Treatment of these infections led to improved motor fluctuations in PD [23]. These studies show the microbes-gut-brain axis, which requires further exploration. Neurokinin B, which often accompanies the onset of rosacea, is also involved in the pathogenesis of PD [24]. For ages, doctors have been using tetracycline in the treatment of rosacea. These drugs offer neuroprotection delaying the onset of PD, as seen in the mouse PD model [25], and this finding is consistently seen in other studies $[18,19]$.

Patients should avoid triggers of flushing, follow sun protection, and proper skincare. Therapeutic interventions for rosacea range from the use of topical metronidazole, topical azelaic acid, and topical doxycycline to laser therapies according to the severity of the disease [16].

\section{Bullous pemphigoid}

Bullous pemphigoid (BP) is an autoimmune bullous disease of the skin, mainly seen in the elderly. The median age of onset is 75 years, more commonly observed in women. Patients suffer from generalized pruritic bullous skin eruptions. This eruption is the result of the body's humoral and cellular response against a component of hemidesmosomes, BP antigen 180 (BP180), and BP antigen 230 (BP230) [26].

The mortality rate of patients with BP seen in eastern and western cohorts is higher than that of the general population [27, 28]. Several co-morbidities accompany elderly BP patients, and neurological disorders are one of them [29]. The mean time interval by which neurological disorder precedes BP is 6.7 years, as observed in one of the systematic reviews [30]. Patients of BP are more likely to develop multiple sclerosis as neurological co-morbidity [29], while other commonly observed are dementia and Parkinson's disease [29]. Studies show that the presence of neurological co-morbidity gives a poor prognosis in patients of BP [27, 29]. Likewise, the presence of PD in patients of BP also increases their mortality [27, 28]. The neurological disorder thus appears as a significant prognostic factor in patients of BP. Many studies calculate PD to be three times more likely to be seen in patients suffering from BP than the general population [29, 30]. A metaanalysis by Lai YC et al. states that the patients of BP are more likely to have a neurological disorder, and 
The revelation of auto-antigens BP180 and BP230 in central nervous system (CNS) apart from the skin discloses the epidemiological relation of BP with neurological diseases [31, 32]. We can postulate a crossreactive immune response between neural and cutaneous antigens of neurodegeneration and neuroinflammation [33]. Circulating immunoglobulin $\mathrm{G}(\mathrm{IgG})$ antibodies were found in a subset of patients of PD directed against BP 180, but their role was doubtful as these anti neural antibodies exhibited no tropism to the skin, and neither did it cause BP like symptoms in them [34]. Psychiatric disorders like bipolar disorders, schizophrenia, personality disorders are also observed more frequently in BP patients, although their risk ratio is lower than the neurological disorders [35]. This association opens the horizon for further research on the role of autoimmunization against neural BP in the pathogenesis of BP.

Physicians should acknowledge the relation of neurological disorder in BP and the subsequent increase in mortality. This issue should be addressed in a therapeutic approach to decrease psychosocial stress.

\section{Melanoma}

Melanoma arises from the melanin-producing cells of the epidermis. The mortality in patients of Parkinson's disease from cancer is significantly lower than that of the general population except for melanoma [36]. The annual new cases of melanoma, in US whites, is predicted to rise from 70,000 in 2007-2011 to 116,000 in 2026-2031. A total of $21 \%$ of this data is attributed to population growth and aging [37].

A large epidemiological study conducted showed that (phase I) patients with PD were 3.8 times more likely to have preexisting melanoma as compared to controls. Whereas in phase II of study, melanoma patients had 4.2 times increased risk of developing PD. Surprisingly, patients who had melanoma without PD were 10.5 times more likely to die from metastatic melanoma compared to patients who had melanoma and PD [38]. Another study of the National Institutes of Health (NIH) Exploratory Trials in PD (NET-PD) Long-term Study 1 cohort also showed a similar risk of malignant melanoma, with a standardized event ratio of 3.6 (95\% confidence interval, 2.2-5.6) [39]. Similarly, another large prospective study of 157,036 patients followed for 14-20 years concluded that the family history of melanoma in 1st-degree relative was associated with a higher risk of PD with a multi-variant relative risk of 1.85 [40].

There are many postulated theories for the association between PD and melanoma. One of them is levodopa therapy, which speculated that levodopa therapy increased the growth and metastatic spread of malignant melanoma in PD [41]. However many trials later, one of them, the deprenyl and tocopherol antioxidative therapy of parkinsonism (DATATOP) clinical trial, clarified the poor link between them, weakening this theory [42]. There is a defect in autophagy in PD, which results in the aggregation of proteins and damaged mitochondria [43]. This defective autophagy is also used by melanoma to escape the immune system [44]. Another theory is the role of $\alpha$-synuclein in PD and melanoma. Under pathological stress, $\alpha$-synuclein accumulates in the dopaminergic neurons. It forms intermediates with dopaquinone and other dopamine metabolites that cause the death of neurons [45]. Melanoma cells also express a high level of $\alpha$-synuclein. These cells produce a low level of melanin due to inhibition of the activity of tyrosine hydroxylase enzyme [46]. Based on these clues, we can assume a possible explanation for an increase in the risk of melanoma in patients of PD. The patients of PD have increased $\alpha$-synuclein in their melanocytes, which decreases melanin synthesis and thus increases the risk of melanoma in them [47]. There is also an increase in the risk of PD with decreasing darkness of hair colour. This observation highlights the role of pigmentation in PD and melanoma. Red hair/fair skin, a phenotype due to loss of function polymorphism in the MC1R gene (melanocortin one receptor gene), is significantly implied in melanoma. The loss of function variants of this gene is also linked to an increased risk of PD [48]. Mutation of Parkin, a tumor suppressor gene located on chromosome 6q25-27, causes young-onset PD [49]. Similarly, the loss of heterozygosity in the chromosome6q25-q27 region is also noted in human cancers, including melanoma [50]. These studies imply the possible genetic association with the simultaneous occurrence of melanoma and PD.

An important role of physicians caring for PD or melanoma is to inform their patients adequately on relevant knowledge of the disease and their associations. They should be advised on skin protection from ultraviolet (UV) rays, especially in lighter skin tones. Physicians should also make the patients cautious about any new or changing pigmented skin lesions [5]. PD and melanoma associations specifically require an acknowledgement among dermatologists, neurologists, oncologists, ophthalmologists, and general practitioners who are directly involved in these patients' care.

\section{Conclusions}

The condition of the skin in patients of PD is often trivialized and overlooked. The primary purpose of our review article was to elucidate the dermatological disorders in PD and explore the linkage in their pathophysiology, genetics, and immunology, focusing on any role of the nervous system in the skin. This knowledge gives a better ground for dermatologists, neurologists, oncologists, and general physicians to advise and care better for their patients and improve their quality of life. Future surgical/nonsurgical treatment options for PD may positively or negatively affect the skin disorders associated with PD. Therefore, further research is needed to establish the robust cause of the increased prevalence of skin 


\section{Additional Information \\ Disclosures}

Conflicts of interest: In compliance with the ICMJE uniform disclosure form, all authors declare the following: Payment/services info: All authors have declared that no financial support was received from any organization for the submitted work. Financial relationships: All authors have declared that they have no financial relationships at present or within the previous three years with any organizations that might have an interest in the submitted work. Other relationships: All authors have declared that there are no other relationships or activities that could appear to have influenced the submitted work.

\section{References}

1. Lebouvier T, Chaumette T, Paillusson S, Duyckaerts C, des Varannes SB, Neunlist M, Derkinderen P: The second brain and Parkinson's disease. Eur J Neurosci. 2009, 30:735-741. 10.1111/j.1460-9568.2009.06873.x

2. E Ray Dorsey, Alexis Elbaz, Emma Nichols, et al.: Global, regional, and national burden of Parkinson's disease, 1990-2016: a systematic analysis for the global burden of disease study 2016. Lancet Neurol. 2016, 17:939-953. 10.1016/S1474-4422(18)30295-3

3. Wu SL, Liscic RM, Kim SY, Sorbi S, Yang YH: Nonmotor symptoms of Parkinson's disease. 2017, 2017:10.1155/2017/4382518

4. Stern MB, Lang A, Poewe W: Toward a redefinition of Parkinson's disease . Mov Disord. 2012, 27:54-60. 10.1002/mds.24051

5. Ravn AH, Thyssen JP, Egeberg A: Skin disorders in Parkinson's disease: potential biomarkers and risk factors. Clin Cosmet Investig Dermatol. 2017, 10:87-92. 10.2147/CCID.S130319

6. Arsic Arsenijevic VS, Milobratovic D, Barac AM, Vekic B, Marinkovic J, Kostic VS: A laboratory-based study on patients with Parkinson's disease and seborrheic dermatitis: the presence and density of malassezia yeasts, their different species and enzymes production. BMC Dermatol. 2014, 14:5. 10.1186/1471-5945-14-5

7. Laurence M, Benito-León J, Calon F: Malassezia and Parkinson's disease. Front Neurol. 2019, 24:758. 10.3389/fneur.2019.00758

8. Amado Y, Patiño-Uzcátegui A, Cepero de García MC, et al.: Seborrheic dermatitis: predisposing factors and ITS2 secondary structure for malassezia phylogenic analysis. Med Mycol. 2013, 51:868-875.

9. Kohn SR, Pochi PE, Strauss JS, Sax DS, Feldman RG, Timberlake WH: Sebaceous gland excretion in parkinson's disease during l-dopa treatment. J Invest Dermatol. 1973, 60:134-136. 10.1111/15231747.ep12682040

10. Martignoni E, Godi L, Pacchetti C et al.: Is seborrhea a sign of autonomic impairment in Parkinson's disease?. J Neural Transm. 1997, 104:1295-1304. 10.1007/BF01294730

11. Tanner C, Albers K, Goldman S, Fross R, Leimpeter A, Klingman J, Van Den Eeden S: Seborrheic dermatitis and risk of future Parkinson's disease. Neurology. 2012, 78:S42.001-S42.001.

12. Ijaz N, Fitzgerald D: Seborrhoeic dermatitis. Br J Hosp Med. 2017, 78:88-91. 10.12968/hmed.2017.78.6.C88

13. Lally A, Casabonne D, Newton R, Wojnarowska F: Seborrheic dermatitis among Oxford renal transplant recipients. J Eur Acad Dermatol Venereol. 2010, 24:561-564. 10.1111/j.1468-3083.2009.03470.x

14. Kustrimovic N, Rasini E, Legnaro M, et al.: Dopaminergic receptors on CD4+ T naive and memory lymphocytes correlate with motor impairment in patients with parkinson's disease. Sci Rep. 2016, 33738. 10.1038/srep33738

15. Neuber K, Kröger S, Gruseck E, Abeck D, Ring J: Effects of pityrosporum ovale on proliferation, immunoglobulin (IgA, G, M) synthesis and cytokine (IL-2, IL-10, IFNY) production of peripheral blood mononuclear cells from patients with seborrhoeic dermatitis. Arch Dermatol Res. 1996, 288:532-536. 10.1007/BF02505250

16. Rainer BM, Kang S, Chien AL: Rosacea: epidemiology, pathogenesis, and treatment. Dermatoendocrinol. 2017, 9:1361574-2017. 10.1080/19381980.2017.1361574

17. Fischer M, Gemende I, Marsch WC, Fischer PA: Skin function and skin disorders in parkinson's disease . I Neural Transm. 2001, 108:205-213. 10.1007/s007020170088

18. Egeberg A, Hansen PR, Gislason GH, Thyssen JP: Exploring the association between rosacea and parkinson disease: a Danish nationwide cohort study. JAMA Neurol. 2016, 73:529-534. 10.1001/jamaneurol.2016.0022

19. Mathieu RJ, Guido N, Ibler E, et al.: Rosacea and subsequent diagnosis for Parkinson's disease: a large, urban, single center, US patient population retrospective study. J Eur Acad Dermatol Venereol. 2017, 32:141-144. 10.1111/jdv.14638

20. Jang YH, Sim JH, Kang HY, Kim YC, Lee ES: Immunohistochemical expression of matrix metalloproteinases in the granulomatous rosacea compared with the non-granulomatous rosacea. J Eur Acad Dermatol Venereol. 2011, 25:544-548. 10.1111/j.1468-3083.2010.03825.x

21. Chung YC, Kim YS, Bok E, Yune TY, Maeng S, Jin BK: MMP-3 contributes to nigrostriatal dopaminergic neuronal loss, BBB damage, and neuroinflammation in an MPTP mouse model of Parkinson's disease. Mediators Inflamm. 2013, 2013:370526.

22. Alexoudi A, Alexoudi I, Gatzonis S: Parkinson's disease pathogenesis, evolution and alternative pathways: a review. Rev Neurol. 2018, 174:699-704. 10.1016/j.neurol.2017.12.003

23. Fasano A, Visanji NP, Liu LW, Lang AE, Pfeiffer RF: Gastrointestinal dysfunction in Parkinson's disease . Lancet Neurol. 2015, 14:625-639. 10.1016/S1474-4422(15)00007-1

24. Karpouzis A, Avgeridis P, Tripsianis G, Gatzidou E, Kourmouli N, Veletza S: Assessment of tachykinin receptor 3' gene polymorphism rs3733631 in rosacea. Int Sch Res Notices. 2015, 2015:469402-10.

25. Lazzarini M, Martin S, Mitkovski M, Vozari RR, Stuhmer W, Bel ED: Doxycycline restrains glia and confers neuroprotection in a 6-OHDA Parkinson model. GLIA. 2013, 61:1084-1100. 10.1002/glia.22496

26. Bernard P, Antonicelli F: Bullous pemphigoid: a review of its diagnosis, associations and treatment . Am J 
Clin Dermatol. 2017, 18:513-528. 10.1007/s40257-017-0264-2

27. Kalinska-Bienias A, Lukowska-Smorawska K, Jagielski P, Kowalewski C, Wozniak K: Mortality in bullous pemphigoid and prognostic factors in 1st and 3rd year of follow-up in specialized centre in Poland. Arch Dermatol Res. 2017, 309:709-719. 10.1007/s00403-017-1772-x

28. Cai SC, Allen JC, Lim YL, Chua SH, Tan SH, Tang MB: Mortality of bullous pemphigoid in Singapore: risk factors and causes of death in 359 patients seen at the national skin centre. Br J Dermatol. 2014, 170:13191326. 10.1111/bjd.12806

29. Lai YC, Yew YW, Lambert WC: Bullous pemphigoid and its association with neurological diseases: a systematic review and meta-analysis. J Eur Acad Dermatol Venereol. 2016, 30:2007-2015. 10.1111/jdv.13660

30. Milani-Nejad N, Zhang M, Kaffenberger J: The association between bullous pemphigoid and neurological disorders: a systematic review. Eur J Dermatol. 2017, 27:472-481. 10.1684/ejd.2017.3066

31. Seppanen A, Autio-Harmainen H, Alafuzoff I, et al.: Collagen XVII is expressed in human CNS neurons . Matrix Biol. 2006, 25:185-188. 10.1016/j.matbio.2005.11.004

32. Kunzli K, Favre B, Chofflon M, Borradori L: One gene but different proteins and diseases: the complexity of dystonin and bullous pemphigoid antigen 1. Exp Dermatol. 2016, 25:10-16. 10.1111/exd.12877

33. Försti AK, Huilaja L, Schmidt E, Tasanen K: Neurological and psychiatric associations in bullous pemphigoid - more than skin deep?. Exp Dermatol. 2017, 26:1228-1234. 10.1111/exd.13401

34. Messingham K A, Aust S, Helfenberger J, et al.: Autoantibodies to collagen XVII are present in Parkinson's disease and localize to tyrosine-hydroxylase positive neurons. J Invest Dermatol. 2016, 136:721-723. 10.1016/j.jid.2015.12.005

35. Forsti AK, Jokelainen J, Ansakorpi H, Seppänen A, Majamaa K, Timonen M, Tasanen K: Psychiatric and neurological disorders are associated with bullous pemphigoid - a nationwide Finnish care register study. Sci Rep. 2016, 6:37125. 10.1038/srep37125

36. Bose A, Petsko GA, Eliezer D: Parkinson's disease and melanoma co-occurrence and mechanisms . J Parkinsons Dis. 2018, 8:385-398. 10.3233/JPD-171263

37. Whiteman DC, Green AC, Olsen CM: The growing burden of invasive melanoma: projections of incidence rates and numbers of new cases in six susceptible populations through 2031. J Invest Dermatol. 2016, 136:1161-1171. 10.1016/j.jid.2016.01.035

38. Dalvin LA, Damento GM, Yawn BP, Abbott BA, Hodge DO, Pulido JS: Parkinson disease and melanoma: confirming and reexamining an association. Mayo Clin Proc. 2017, 92:1070-1079. 10.1016/j.mayocp.2017.03.014

39. Constantinescu R, Elm J, Auinger P, et al.: Malignant melanoma in early-treated parkinson's disease: the NET-PD trial. Mov Disord. 2014, 29:263-265. 10.1002/mds.25734

40. Gao X, Simon KC, Han J, Schwarzschild MA, Ascherio A: Family history of melanoma and parkinson disease risk. Neurology. 2009, 73:1286-1291. 10.1212/WNL.0b013e3181bd13a1

41. Sandyk R: Accelerated growth of malignant melanoma by levodopa in Parkinson's disease and role of the pineal gland. Int J Neurosci. 1992, 63:137-140. 10.3109/00207459208986663

42. Constantinescu R, Romer M, Kieburtz K, et al.: Malignant melanoma in early Parkinson's disease: the DATATOP trial. Mov Disord. 2007, 22:720-722. 10.1002/mds.21273

43. Pan T, Kondo S, Le W, Jankovic J: The role of autophagy-lysosome pathway in neurodegeneration associated with Parkinson's disease. Brain. 2008, 131:1969-1978. 10.1093/brain/awm318

44. Eskelinen EL, Saftig P: Autophagy: a lysosomal degradation pathway with a central role in health and disease. Biochim Biophys Acta. 2009, 1793:664-673. 10.1016/j.bbamcr.2008.07.014

45. Xu J, Kao SY, Lee FJ, Song W, Jin LW, Yankner BA: Dopamine-dependent neurotoxicity of alpha-synuclein: a mechanism for selective neurodegeneration in Parkinson disease. Nat Med. 2002, 8:600-606. 10.1038/nm0602-600

46. Peng X, Peng XM, Tehranian R, Dietrich P, Stefanis L, Perez RG: Alpha-synuclein activation of protein phosphatase 2A reduces tyrosine hydroxylase phosphorylation in dopaminergic cells. J Cell Sci. 2005, 118:3523-3530. 10.1242/jcs.02481

47. Tessari I, Bisaglia M, Valle F, Samorì B, Bergantino E, Mammi S, Bubacco L: The reaction of alphasynuclein with tyrosinase: possible implications for Parkinson disease. J Biol Chem. 2008, 283:16808-16817. 10.1074/jbc.M709014200

48. Tell-Marti G, Puig-Butille JA, Potrony M, et al.: The MC1R melanoma risk variant p.R160W is associated with Parkinson disease. Ann Neurol. 2015, 77:889-894.

49. Cesari R, Martin ES, Calin GA, et al.: Parkin, a gene implicated in autosomal recessive juvenile parkinsonism, is a candidate tumor suppressor gene on chromosome 6q25-q27. Proc Natl Acad Sci U S A. 2003, 100:5956-5961. 10.1073/pnas.0931262100

50. Millikin D, Meese E, Vogelstein B, Witkowski C, Trent J: Loss of heterozygosity for loci on the long arm of chromosome 6 in human malignant melanoma. Cancer Res. 1991, 51:5449-5453. 